\title{
Dynamic demand estimation for an AMoD system in Paris
}

\section{Conference Paper}

\section{Author(s):}

Hörl, Sebastian; Balać, Miloš; Axhausen, Kay W. (D)

Publication date:

2019-06-10

Permanent link:

https://doi.org/10.3929/ethz-b-000354843

Rights / license:

In Copyright - Non-Commercial Use Permitted

Originally published in:

https://doi.org/10.1109/IVS.2019.8814051 


\title{
Dynamic demand estimation for an AMoD system in Paris
}

\author{
Sebastian Hörl, Milos Balac, and Kay W. Axhausen
}

\begin{abstract}
A simulation framework is presented that equilibrates a given automated taxi fleet with (a) consistent prices to provide the service and (b) customer behaviour that reacts to costs and level of service alike. In a first attempt, a hypothetical AMoD service within the highway ring of Paris is considered. The "dynamic demand" case yields a demand of around 1.2M trips per day for such a service at the optimal fleet size of $25 \mathrm{k}$ vehicles. This number is considerably lower than $2.3 \mathrm{M}$ trips that potentially could be served in a "static maximum demand" case which has often been used as a basis for previous fleet sizing studies. While the authors acknowledge a multitude of assumptions that constitute the present model, clear pathways to its improvement, methodologically and datawise, are provided.
\end{abstract}

\section{INTRODUCTION}

Automated vehicles have become an active field of research in transport planning over the recent years. While technology is developing with quick pace, more and more insightful studies considering the impact of automated vehicles on the transport system are conducted. Especially the case of Autonomous Mobility on Demand (AMoD), i.e. automated taxis, is discussed vividly: While a shift towards the sharing economy with less private vehicle ownership and more service-oriented mobility promises to reduce the number of vehicles on the roads and offer mobility to previously excluded user groups, negative impacts are to be expected as well. A usual assumption is that, although less vehicles would be on the roads, they would drive more than before. The vehicle kilometers travelled (VKT) would increase because of empty rides to the customers, redistribution of vehicles in the network or even induced demand if travelling gets so convenient. This would come with the negative effects that go along with increasing VKT such as faster deterioration of infrastructure and higher emissions.

The contribution of this paper to the ongoing discussion around automated mobility is twofold: To the best knowledge of the authors, the study provides a first estimate of an $\mathrm{AMoD}$ fleet size for the city of Paris. By that means a novel agentbased transport model for the Île-de-France region is introduced. Furthermore, the work exceeds previous attempts of fleet size estimates by providing a methodology that does not only consider a static trip demand. In this study a dynamic demand that is sensitive to the cost of transportation and level of service must be served by a properly sized AMoD fleet. The presented methodology is based on an extended study that has been conducted for Zurich, Switzerland [1].

\section{BACKGROUND}

Control and management of automated vehicle fleets has become an active field of research over the past five years.
The following paragraphs shall give a brief overview of past simulation studies that have been performed for various use cases around the world.

One of the first large-scale simulations was performed in [2] for Singapore. The study finds that the whole transport demand of the city could be covered by one third of today's vehicle fleet if it entirely would consist of automated singleoccupancy vehicles.

Subsequently, a series of studies have been performed for the case of Austin, Texas. In [3] a grid-based simulation for the city is introduced. For an artificial demand based on real-world trip generation rates and randomly assigned destinations it is found that Austin's demand in private car trips could be served by an automated vehicle fleet that is reduced by $90 \%$ compared to today. The use case is further extended in [4], where an electric charging infrastructure is assumed. Further studies introduce a more detailed demand for the scenario, based on static trips from the regional household travel survey (HTS). [5] introduces congestion to the simulation and finds that this has a strong impact on fleet size. In [6] a choice model is applied, though in a postprocessing step. After a detailed daily travel demand from HTS is simulated using an approach that had been applied to the canton of Zurich before [7], a discrete choice model is fed with information about travel and waiting times to analyze potential mode shares in Austin. The utility function is entirely based on literature, no model is estimated from stated preference (SP) data. Finally, [8] extends the Austin case with a ride-sharing component and finds that it could reduce wait times for the customers and mitigate the increase of VKT (vehicle kilometers travelled) due to empty rides. However, customer preferences for the service are not taken into account.

For the case of Berlin, [9] use a static travel demand from the regional HTS to create a MATSim [10] (see below) simulation with automated taxis. All car trips within the city boundaries are replaced by the service, leading to a scenario where one tenth of all vehicles could be replaced if every agent in the simulation was to use the service with acceptable wait times. In the same scenario [11] find that also allowing public transport users leads to a linear increase in needed fleet size. Finally, [12] introduce congestion to the simulation showing that without significant gains in road capacity due to automation a fleet of automated taxis serving all of the city's demand would worsen congestion dramatically. On the other hand, automated vehicles are found to be likely to mitigate parking search problems in the city [13].

For Zurich, [14] shows that under ideal flow conditions a fleet size of around 7000 to 14000 automated taxis would 
be able to serve the mobility demand of the city. Using a detailed agent-based daily travel demand from HTS data, it is shown that the dispatching strategy has a large impact on the performance of the fleet. Extended work [1] combines this simulation with a detailed model of costs for automated mobility [15]. A choice model for conventional and automated modes of transport alike is estimated from a large-scale stated preference survey in the canton of Zurich and added dynamically to the simulation. The study constitutes the first simulation in which a closed cycle between simulation of demand and supply is able to not only estimate what fleet size would be able to serve a certain demand, but also for which fleet size and service characteristics customers would be willing to pay.

A similar approach is shown in [16] for a not further specified European city. Not using an agent-based transport model, the simulation is based on trip generation rates, which are in turn dependent on a discrete choice model that is fed with travel and waiting times from a previous simulation of the generated trips. Hence, equilibrium of supply and demand can be achieved.

Further simulations based on Aimsum have been performed for Munich [17], [18] where HTS data and realworld car-sharing data is used to establish a demand that is to be covered by automated taxis. [19] introduce another simulation platform for Lisbon, where HTS data is used in a static way, but where the choice for various automated mobility services is determined by a heuristic model based on expert opinion.

For France, a number of studies can be mentioned. [20] propose a simulation framework of automated busses in Palaiseau close to Paris. Demand is static and based on tripgeneration rates at well-defined spots in the system. The simulation is extended in [21], where a choice model is introduced to establish a dynamic demand. Utilities for the choice model are based on literature values.

A first simulation with automated vehicles in Paris is presented in [22]. Based on census and HTS data, similar to the paper at hand, the characteristics of a static fleet of 17000 vehicles are examined serving a detailed dynamic demand based on a classic MATSim simulation. No SP data is used, but a heuristic that, based on literature, models the attractivity of an automated taxi service for different sociodemographic and income-based groups. As a first study in the field the impact of such a fleet on sociodemographic groups is studied in detail. The framework is further applied to the city of Rouen [23], where a fleet sizing given diverse user group preferences is performed. It is found that user preferences may have a strong impact on the utilization of such a service.

\section{Simulation FrameWORK}

As can be seen from the previous section, agent-based models are commonly applied to the simulation of AMoD systems because of their ability to model individual trips and interaction of entities such as travellers and taxis. For the study at hand the agent-based transport simulation framework MATSim has been chosen. In the next section a short introduction to MATSim will be given, followed by a more detailed discussion of a number of extensions that have been made to the system.

\section{A. MATSim}

A typical MATSim simulation needs a population of travellers as an input. It contains a large number of traveller agents with sociodemographics and detailed daily plans. An agent may have multiple activities throughout one day that should be conducted at a certain time for a certain duration. Those activities are connected by trips, which are assigned a certain mode of transport.

For the simulation of road-based traffic MATSim provides a network simulation component which works in a timestepand queue-based manner. Depending on when agents enter certain road segments they may be delayed due to emergent traffic jams or get slowed down because of busses or other vehicles blocking the road.

For the present work, mainly the convenient data structures of MATSim, as well as the network simulation are used. On top of that, standard MATSim also provides an "evolutionary learning loop" in which agents adapt their daily plans to the given transport system and other agents' decisions. In this work, a different approach is used which applies a discrete mode choice model directly to modify agents' plans.

\section{B. Discrete mode choice model}

In standard MATSim, agents learn by applying random modifications to their current plan, assigning a score as soon as it has been simulated in the next iteration of the simulated day and comparing this score to previously tested plans. By always keeping (and selecting between) a number of plans as the basis for the next iteration and by always removing the worst ones, each agent optimizes its own plan until a stochastic user equilibrium is reached. Unfortunately, this process can be rather slow since improvements happen purely by chance and the probability that highly unlikely plans are generated for testing (such as walking to work for $50 \mathrm{~km}$ ) is high.

For these and other reasons, work has recently been put into using existing discrete choice models within the MATSim loop to make decisions [24], [25]. The way the system works (Figure 1) is that choice dimensions such as travel times, waiting times and others are estimated from the latest network simulation and/or previous iterations. At the end of each cycle a configurable share of agents applies a given mode choice model to their plans. Subsequently, the state of the transport system will change and different decisions may be taken in the next iterations. Ultimately, mode shares stabilize and go into equilibrium with the traffic conditions in the network.

The framework makes it easy to switch between different model formulations. Currently, utility functions can be used in a best-response context or as part of a multinomial logit model[26]. Also, a purely random selection of choice alternatives can be configured. In any case, the choice set available to an agent is restricted by a number of constraints, 


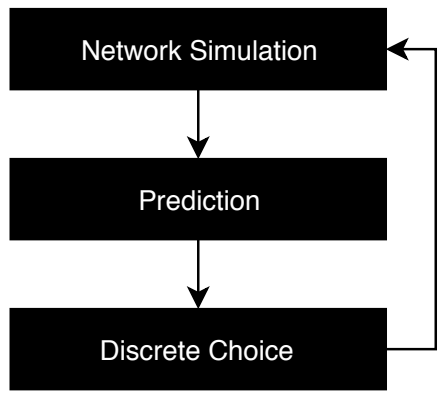

Fig. 1. Iterative structure of the simulation: First, the synthetic population is simulated (in combination with automated taxis). Second, predictions for the updated choices of the travellers are made. Third, based on the prediction a mode choice model is used to arrive at new daily travel plans.

the most important being the "vehicle continuity constraint". It defines that an agent is only allowed to choose a certain mode of transport if the respective vehicle has been moved to the origin of the trip in question. For purely trip-based models, the considered choice sets consist of single mode options for a specific trip, but tour-based or whole daybased choice sets can be generated to allow for richer, but computationally more expensive decisions.

\section{Simulation of automated vehicles}

For the simulation of automated vehicles an extension to MATSim [27], [28] is used. Each vehicle is simulated independently in the network. A global dispatching component controls the movement of fleet vehicles and manages the assignment of requests. Those requests are created online, i.e. as soon as a traveller wants to depart at a certain location. The dispatcher then uses a heuristic algorithm developed in [9]: In case there are more unoccupied vehicles than pending requests in the system the closest vehicle to any incoming request is assigned to it. If the amount of requests exceeds the amount of available vehicles a vehicle is assigned to its closest request as soon as it becomes available. The vehicle then drives through the network to pick up the customer and subsequently moves the passenger to its destination. No reassignments of requests and vehicles while approaching the customer are taking place in this approach.

It must be noted that the heuristic approach is optimized for simulation speed, while other algorithms may produce better results. In fact, previous studies [14] have shown that more computationally expensive algorithms may outperform the heuristic, especially with regards to specific objectives such as minimizing overall customer waiting time or minimizing fleet empty mileage. Many of such algorithms have been developed in the AMoDeus framework [29], which adds an open easy-to-use fleet management benchmarking layer on top of the code used here.

\section{SyNThETIC POPUlation OF PARIS}

To perform the proposed fleet sizing experiments, the simulation framework needs to operate on a population of synthetic agents. The following section details how such a population is set up for the Île-de-France region, which surrounds the city of Paris. Afterwards, further modifications for future scenarios of automated mobility are described.

\section{A. Baseline Scenario}

The novel synthetic agent population of Paris consists of individual persons, aggregated to households, which have a rich set of individual daily activity schedules. It is known where people live, where they work, where they perform leisure or shopping activities, and when and in which order they want to perform them.

The population is created in a multi-step process:

- For France, a large sample of census data ${ }^{1}$ is available for 2015. In the first synthesis step, the households contained in this data set are filtered such that only residents of the Île-de-France region remain. Using the provided weights, a full population for the region is sampled, which includes all relevant socio-demographic attributes such as age, gender, employment and more.

- In order to synthesize daily activity chains for those agents, the regional Household Travel Survey is used (Enquête globale de transport ${ }^{2}$ ). It contains the activity chains of 35,175 respondents including information about their sociodemographics. Using these attributes, activity chains are attached to all synthetic agents by statistical matching.

- Home locations of all households are known from the census data, but only on the basis of IRIS zones (see Figure 2). Therefore, a discrete location within the respective zone is sampled for each household. For primary activities (like work and education) commuter matrices $^{3}$ are used to sample destination zones proportionately given the homes of the agents. For all other activities (shopping, leisure, errand) locations are sampled at random from the French Enterprise Census 2016 (Base permanente des équipements ${ }^{4}$ ) such that they are consistent with modes and travel times in each agent's daily plan.

- Finally, the synthetic population is converted to the MATSim data format to be used by the simulation.

To model the supply side two major data sources are used. First, OpenStreetMap data ${ }^{5}$ is used to create a road network of Île-de-France. Second, public transit schedules, which are published on a regular basis for the whole region ${ }^{6}$, are integrated. These steps are performed using existing tools [30] in the MATSim ecosystem. It is worth noting that all used data sets, except the HTS, are publicly available.

Subsequently, the population is simulated in MATSim in combination with the discrete choice extension. The choice

\footnotetext{
${ }^{1}$ https://www.insee.fr/fr/statistiques/3625223? sommaire $=3558417$

${ }^{2} \mathrm{http}$ ///www.driea.ile-de-france.developpement-durable.gouv.fr/enqueteglobale-de-transport-r18.html

${ }^{3}$ https://www.insee.fr/fr/statistiques/3566008?sommaire $=3558417$

${ }^{4}$ https://www.data.gouv.fr/fr/datasets/base-permanente-des-equipements$1 /$

${ }^{5}$ https://download.geofabrik.de/europe/france/ile-de-france.html

${ }^{6} \mathrm{https} / / /$ opendata.stif.info/explore/dataset/offre-horaires-tc-gtfsidf/information/
} 


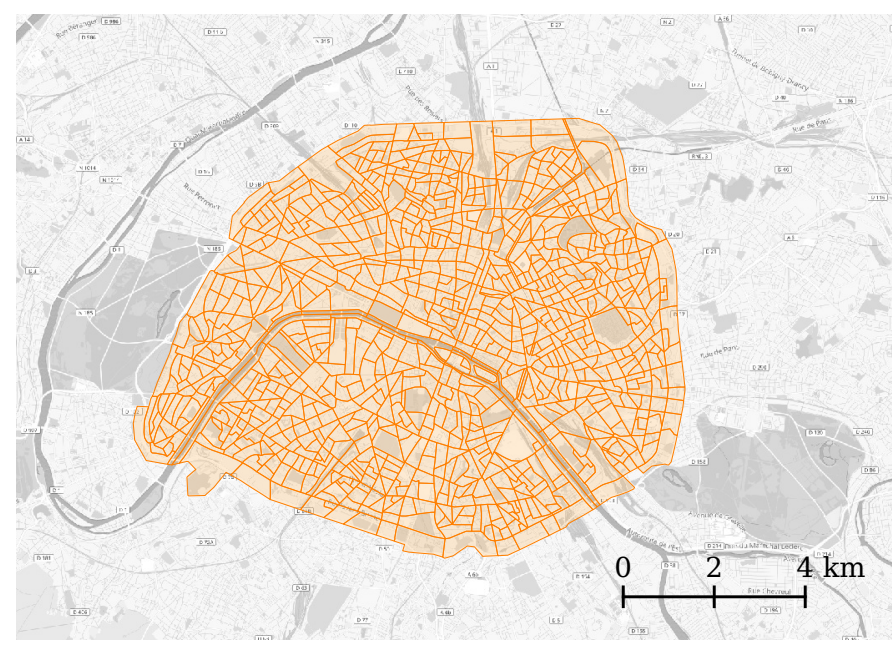

Fig. 2. Map of Paris with the IRIS zoning system. The colored area is used as the operating area in this study. (Map: OpenStreetMap / Wikimedia)

TABLE I

PARAMETERS OF THE DISCRETE MODE CHOICE MODEL

\begin{tabular}{l|lrl}
\hline \hline Car & $\alpha_{\text {car }}$ & 1.35 & \\
& $\beta_{\text {travelTime,car }}$ & -0.0667 & {$\left[\mathrm{~min}^{-1}\right]$} \\
\hline Public Transport & $\alpha_{\mathrm{pt}}$ & 0.0 & \\
& $\beta_{\text {numberOfTransfers }}$ & -0.17 & \\
& $\beta_{\text {inVehicleTime }}$ & -0.017 & {$\left[\mathrm{~min}^{-1}\right]$} \\
& $\beta_{\text {transferTime }}$ & -0.0484 & {$\left[\mathrm{~min}^{-1}\right]$} \\
& $\beta_{\text {accessEgressTime }}$ & -0.0804 & {$\left[\mathrm{~min}^{-1}\right]$} \\
\hline Bike & $\alpha_{\text {bike }}$ & 0.1 & \\
& $\beta_{\text {travelTime,bike }}$ & -0.15 & {$\left[\mathrm{~min}^{-1}\right]$} \\
& $\beta_{\text {age,bike }}$ & -0.0496 & {$\left[\mathrm{a}^{-1}\right]$} \\
\hline Walk & $\alpha_{\text {walk }}$ & 1.43 & \\
& $\beta_{\text {travelTime,walk }}$ & -0.09 & {$\left[\mathrm{~min}^{-1}\right]$} \\
\hline Others & $\beta_{\text {cost }}$ & -0.206 & {$[\mathrm{EUR}$} \\
& $\lambda$ & -0.4 & \\
& $\theta_{\text {averageCrowflyDistance }}$ & 40 & {$[\mathrm{~km}]$} \\
\hline Calibration & $\theta_{\text {parkingSearchPenalty }}$ & 4 & {$\left[\mathrm{~min}^{-1}\right.$} \\
& $\theta_{\text {accessEgressWalkTime }}$ & 4 & {$[\mathrm{~min}]$} \\
\hline \hline
\end{tabular}

model formulation is documented in Equations 1 to 4 with the parameter values given in Table I. Expressions denoted as $x$ describe choice variables while $\theta$ and $a$ denote calibration parameters and agent-specific constants, respectively.

$$
\begin{aligned}
u_{\mathrm{car}}(x) & =\alpha_{\mathrm{car}} \\
& +\beta_{\text {travelTime,car }} \cdot x_{\text {travelTime,car }} \\
& +\beta_{\text {travelTime,car }} \cdot \theta_{\text {parkingSeachPenalty }} \\
& +\beta_{\text {travelTime,walk }} \cdot \theta_{\text {accessEgressWalkTime }} \\
& +\beta_{\text {cost }} \cdot\left(\frac{x_{\text {crowflyDistance }}}{\theta_{\text {averageDistance }}}\right)^{\lambda} \cdot x_{\text {cost,car }} \\
u_{\mathrm{pt}}(x) & =\alpha_{\mathrm{pt}} \\
& +\beta_{\text {numberOfTransfers }} \cdot x_{\text {numberOfTransfers }} \\
& +\beta_{\text {inVehicleTime }} \cdot x_{\text {inVehicleTime }} \\
& +\beta_{\text {transferTime }} \cdot x_{\text {transferTime }} \\
& +\beta_{\text {accessEgressTime }} \cdot x_{\text {accessEgressTime }} \\
& +\beta_{\text {cost }} \cdot\left(\frac{x_{\text {crowflyDistance }}}{\theta_{\text {averageDistance }}}\right)^{\lambda} \cdot x_{\text {cost }, \mathrm{pt}}
\end{aligned}
$$

$$
\begin{aligned}
u_{\text {bike }}(x) & =\alpha_{\text {bike }} \\
& +\beta_{\text {travelTime,bike }} \cdot x_{\text {travelTime,bike }} \\
& +\beta_{\text {age,bike }} \cdot \max \left(0, a_{\text {age }}-18\right) \\
u_{\text {walk }}(x) & =\alpha_{\text {walk }} \\
& +\beta_{\text {travelTime,walk }} \cdot x_{\text {travelTime,walk }}
\end{aligned}
$$

Unfortunately, at the time of writing, the authors do not have access to an extended version of the household travel survey which includes geo-coded start and end locations of observed trips. Since without that information no unchosen alternatives can be examined, no specific model parameters for the Île-de-France region could be estimated. Instead, previously presented parameters and models for the city of Zurich [25], [1] have been used as starting values. The model parameters (with only slight modifications necessary) have then been calibrated such that a number of distributions in the simulation match well the observed reference data: travel times and distances for each mode, mode shares in total, by distance classes and by time of day. Therefore, all $\alpha$ and $\beta$ parameters in the model are initially estimated model parameters that have been adapted to the agent population of Paris. The strongest modifications have been applied to $\beta_{\text {cost }}$, which determines the cost sensitivity of the population. As a first sanity check it can be stated that the value of time in the calibrated model (calculated as $\beta_{\text {travelTime,car }} / \beta_{\text {cost }}$ ) of around $19.40 \mathrm{EUR} / \mathrm{h}$ falls well into the range that has been reported previously for Île-de-France [31].

While a thorough presentation of the validation results for the synthetic population and the choice model exceeds the scope of this paper, we would like to refer the reader to upcoming work [32], which will detail the steps briefly mentioned above and provide more in-depth comparison with reference data.

\section{B. Future Scenarios}

The aim of the future scenarios is to obtain an idea about the demand for AMoD travel in Paris. For that purpose, first an operating area needs to be chosen. For the sake of simplicity, in this research, only the city area of Paris within the highway ring is served by a fleet of automated single-occupancy taxis (see Figure 2). Future studies may be based on a more sensible choice of operating area as will be explained below. Furthermore, our simulations assume that the vehicles are electric, which has an impact on the cost of the service.

Three experiments are set up. In the first experiment, a best-response model is configured in such a way that all trips that can be served by the automated taxi service must be served by it. That means regardless of waiting times or travel costs, any trips in the daily plans of all travellers that are not otherwise constrained are converted to the AMoD transport mode. Obviously, the experiment assumes that the service is so attractive that it is used in any possible case. The simulation yields therefore an estimate of the fleet size that is needed to serve the "maximum static demand scenario". 
Second, the simulation is run with a multinomial logit model with the additional AMoD transport mode. The parameters of the utility function are derived from existing parameters for public transport since at the time of conducting the experiments no stated choice data or similar is available to to the authors. The utility function is defined as follows:

$$
\begin{aligned}
u_{\mathrm{AMoD}} & =\alpha_{\mathrm{pt}}+\beta_{\text {travelTime } \mathrm{pt}} \cdot x_{\text {travelTime,av }} \\
& +\beta_{\text {transferTime, } \mathrm{pt}} \cdot x_{\text {waitingTime,av }} \\
& +\beta_{\text {cost }} \cdot p_{\mathrm{av}} \cdot x_{\text {networkDistance,av }}
\end{aligned}
$$

The waiting times are based on moving average estimates over 10 iterations. Spatially, they are estimated on the basis of IRIS zones. Here, $p_{a v}$ denotes the price per kilometre, which is fixed to various values in a number of experiments. The experiment therefore has the purpose to explore the resulting demand in the system given a dependency of the choice behaviour on the level of service, a fixed fleet size and a fixed price level.

The third experiment makes use of a detailed cost model of automated mobility [15]. It has been successfully applied to the case of Zurich, Switzerland and has recently been applied to a range of cities worldwide [33]. Depending on the fleet utilization, empty distance, fleet sizes, among other components, the cost model calculates a passenger price per kilometre that covers the expenses of maintaining the service. In the present simulation setup those prices are calculated in each iteration, while a moving average of the previous 10 iterations is fed into the choice model as described in Equation 5. The cost model itself is specified by maintenance costs, interest costs and a multitude of additional factors. Unfortunately, to date, no specific assumptions for Paris are available. Therefore, to present the methodology for a fully dynamic demand estimate, the model specification for Berlin is used, which should be closest to the case of Paris among the available city-specific parameter sets. In the present case, the cost model is configured such that the service should always have zero net costs, i.e. no profit margin is included.

It is important to mention that in all experiments trips that are performed using the "walk" or "bike" transport mode in the baseline scenario are never converted to AMoD trips. Furthermore, constant travel times within 5 minute bins on all network links are enforced as measured from the baseline scenario. Therefore, effects in travel time changes due to fewer or more vehicles on the road are deliberately ignored here. This way, we avoid to motivate additional assumptions on flow efficiency effects of automated vehicles in the current stage of this research.

All simulations are run using a $10 \%$ sample of the full agent population. This way, feasible simulation times of around $5 \mathrm{~h}$ per simulation on a modern cluster are achieved.

\section{RESULTS}

The "maximum demand scenario" yields a number of $2.3 \mathrm{M}$ trips that must be served by the AMoD fleet. This number can be compared to the waiting time-dependent cases as depicted in Figure 3. The demand, that is attracted once

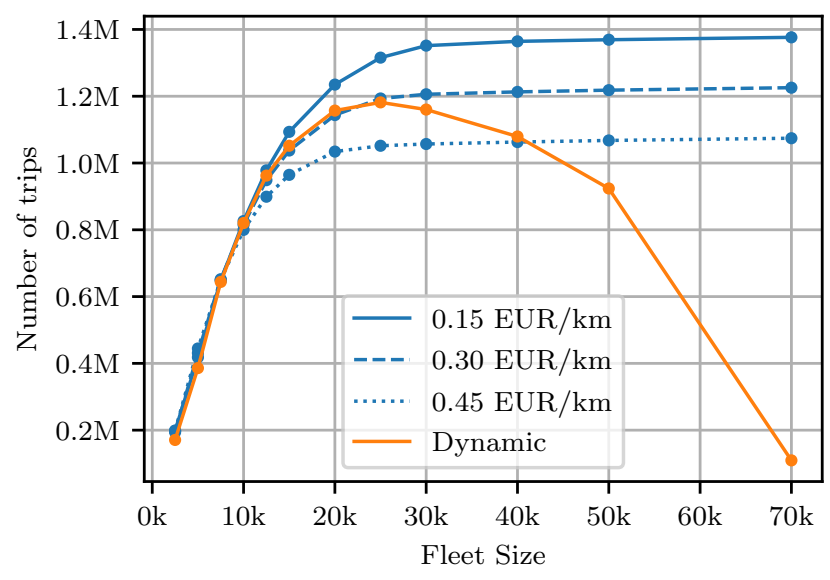

Fig. 3. Dependency of the number of trips done using the AMoD service on fleet size. In the fixed price cases (blue) low fleet sizes lead to high waiting times and low demand, in the dynamic price case (red), high prices for large fleet sizes lead to a demand maximum at around 25k vehicles.

the choice model is in place and waiting time is considered, never reaches this maximum value. In fact, with a maximum of around $1.4 \mathrm{M}$ trips it is only slightly more that half the number of possible trips that could be converted to the AMoD transport mode.

The dependency of demand on fleet size is as expected: With increasing fleet sizes more demand is attracted until it goes into saturation. The low number of trips for small fleet sizes are a direct consequence of the poor waiting times that are produced by smaller AMoD fleets. Also, the travellers' reaction to different prices that are imposed on AMoD travels makes sense: The lower the price, the more demand is attracted in these simulations.

Finally, in the last set of simulations the price is not predefined but calculated from the cost model. The "dynamic pricing" graph in Figure 3 shows that there is a demand maximum if level of service and prices are considered: Larger fleet sizes lead to higher costs and, therefore, to less demand. In these simulations we arrive at an optimal fleet size of around $25 \mathrm{k}$ vehicles which would be able to attract around 1.2M trips during one day. This is one half of all trips that would structurally be possible.

Since the prices, as the waiting times, are endogenous in these simulations, it is interesting to have a look at them, too. Figure 4 shows that there is a price minimum at around $10 \mathrm{k}$ vehicles. Still, at this fleet size already a fair share of demand is lost due to high waiting times. Decreasing the fleet size further even leads to higher prices because of low demand.

The price at the demand optimal fleet size of around $25 \mathrm{k}$ vehicles is around 0.27 EUR per passenger kilometer. In comparison ${ }^{7}$ a price of $0.3 \mathrm{EUR} / \mathrm{km}$ is listed as the cheapest full cost per vehicle kilometer for car ownership in France. Therefore, such a service could be highly attractive for

\footnotetext{
${ }^{7}$ https://www.largus.fr/actualite-automobile/prk-2018-ce-que-vous-coutereellement-votre-voiture-en-2018-8987391.html
} 


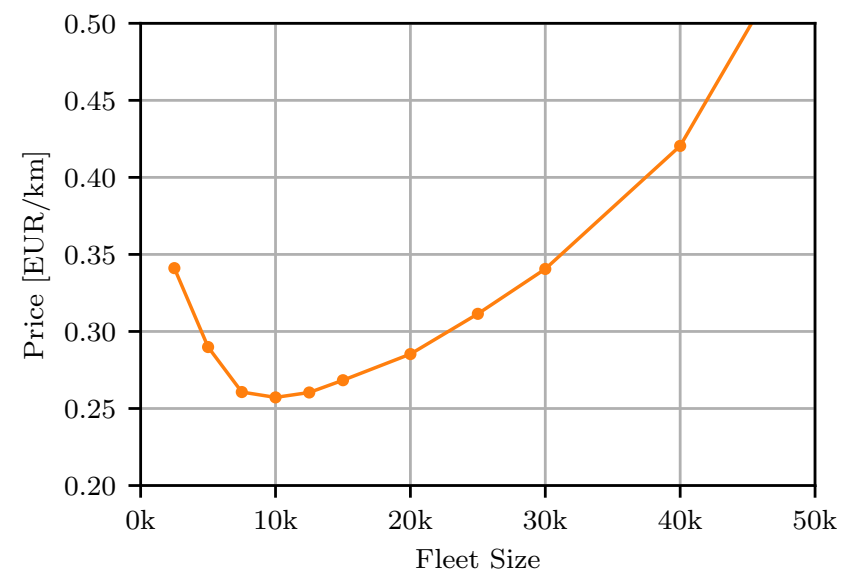

Fig. 4. Dependency of price on AMoD fleet size for the dynamic price case. High fleet sizes lead to high investment costs and therefore high prices per passenger kilometer. However, very low fleet sizes lead to higher prices due to smaller demand.

today's car users if their travels are mainly confined to the proposed operating area. Unsurprisingly, today's minimum taxi fare of $1.07 \mathrm{EUR} / \mathrm{km}^{8}$ in the city exceeds the AMoD tarif by far.

\section{DISCUSSION}

While the results presented above are able to provide a first idea of how a fleet of automated taxis in Paris could look like, they are by no means definitive. Three important components of the simulation can be improved easily, given the necessary data becomes available:

- First, the cost model of automated mobility for the case of Berlin is used in this research as it seems to be closest to Paris among those cities that have been assessed to far. Applying the methodology of [15] explicitly for the case of Paris will yield a more consistent picture of customer prices.

- Second, the choice model and its parameters are derived from a model for Zurich due to the lack of a discrete choice model that is compatible with the choice dimensions provided by MATSim and Île-de-France data. A specifically estimated choice model for the region or a spatially detailed version of the Enquête globale de transport to conduct such an estimation would help improve the consistency of the behavioural component of our model.

- Third, the ideal case would be a discrete choice model including AMoD services and the attitudes of the Îlede-France population towards such an offer.

For the sake of brevity, the analysis in this paper is restricted to the interplay of (revenue neutral) prices and the AMoD demand. Of course, our simulation framework allows the analysis of a multitude of additional dimensions such as vehicle replacement rates, empty distances, overall

\footnotetext{
${ }^{8}$ https://www.service-public.fr/professionnelsentreprises/vosdroits/F22127
}

driven distance, emissions, gains and losses in travel time, even gains and losses in (utility-based) welfare on a peragent basis. This also makes it possible to analyse the impact of an AMoD service for specific sociodemographic groups. Especially the analysis of mode shifts will become important, since a large share of demand in the presented simulations is generated through former public transport users.

The framework allows to introduce a manifold of additional scenarios and to answer respective questions:

- The financial aspects of an AMoD service can be examined. How do specific profit rates or subsidies influence the system?

- Instead of door-to-door travels the AMoD fleet can be simulated as feeder service for the commuter rail and metro network [34]. How would this influence the use of bus services in the city? Would this have impact on the elderly or physically disabled who use such services to avoid inaccessible metro stations?

- Currently the framework is being extended to allow for limited pick-up and drop-off spaces in the network as well as at public transit stations. Will spatial constraints become an issue for the feasibility of a large-scale AMoD service?

- More intelligent dispatching (and redistribution) algorithms can be tested. Looking at previous studies the authors expect a strong effect on the dynamic demand depending on whether an empty-distance-minimizing dispatcher is used or one that tries to distribute waiting times equally in the operating area. While comparative studies in static demand cases have been conducted [14], there is no study to the knowledge of the authors that has assessed different fleet operational strategies under the presence of customer behaviour.

- Different scenario diameters can be defined: Currently, the highway ring of Paris serves as an (computationally feasible) example, which lacks political as well as land-use considerations. For instance, neither the main business district La Défense is included in the scenario, nor are the two main airports Charles-deGaulle and Orly. Many outer neighborhoods of the city are neglected, where, for instance, feeder services to the RER commuter rail could be of benefit to the local population. A further interesting future path to explore lies therefore in questions of land use policy and urban development. For both fields the model at hand could yield valuable inputs.

\section{CONCLUSION}

To conclude, we want to focus on two main insights. First, we show that taking into account traveller behaviour in demand estimation for an AMoD service makes a big difference in the results. The dynamic behaviourally consistent demand is only half the maximal possible number of trips.

Second, we conclude that given our assumptions an AMoD fleet could operate within the highway ring of Paris with around $25 \mathrm{k}$ vehicles at a price of $0.27 \mathrm{EUR} / \mathrm{km}$, which is low compared to the full cost of using one's own private vehicle 
in Paris. This result confirms the findings of previous studies that an AMoD fleet is indeed an economical alternative to today's private car ownership.

Our discussion provides an incomplete list of potential future studies that could be conducted using the framework at hand. While many of the assumptions in the model to date are best guess, there lies great potential in improving it through the various ways described above.

\section{REFERENCES}

[1] S. Hörl, F. Becker, T. Dubernet, and K. W. Axhausen, "Induzierter Verkehr durch autonome Fahrzeuge: Eine Abschätzung," Bundesamt für Strassen (ASTRA), Ittingen, final report for SVI 2016/001, 2018.

[2] K. Spieser, K. Treleaven, R. Zhang, E. Frazzoli, D. Morton, and M. Pavone, Toward a Systematic Approach to the Design and Evaluation of Automated Mobility-on-Demand Systems: A Case Study in Singapore. Cham: Springer International Publishing, 2014, pp. 229245.

[3] D. J. Fagnant and K. M. Kockelman, "The travel and environmental implications of shared autonomous vehicles, using agent-based model scenarios," Transportation Research Part C: Emerging Technologies, vol. 40, pp. 1 - 13, 2014

[4] T. D. Chen, K. M. Kockelman, and J. P. Hanna, "Operations of a shared, autonomous, electric vehicle fleet: Implications of vehicle \& charging infrastructure decisions," Transportation Research Part A: Policy and Practice, vol. 94, pp. 243 - 254, 2016.

[5] M. W. Levin, K. M. Kockelman, S. D. Boyles, and T. Li, "A general framework for modeling shared autonomous vehicles with dynamic network-loading and dynamic ride-sharing application," Computers, Environment and Urban Systems, vol. 64, pp. 373 - 383, 2017.

[6] J. Liu, K. M. Kockelman, P. M. Boesch, and F. Ciari, "Tracking a system of shared autonomous vehicles across the austin, texas network using agent-based simulation," Transportation, vol. 44, no. 6, pp. 1261-1278, Nov 2017.

[7] Bösch, Patrick M. and Ciari, Francesco and Axhausen, Kay W., "Autonomous vehicle fleet sizes required to serve different levels of demand," vol. 2542, no. 4, pp. 111 - 119, 2016.

[8] D. J. Fagnant and K. M. Kockelman, "Dynamic ride-sharing and fleet sizing for a system of shared autonomous vehicles in austin, texas," Transportation, vol. 45, no. 1, pp. 143-158, Jan 2018.

[9] J. Bischoff and M. Maciejewski, "Simulation of City-wide Replacement of Private Cars with Autonomous Taxis in Berlin," Procedia Computer Science, vol. 83, pp. 237 - 244, 2016.

[10] A. Horni, K. Nagel, and K. W. Axhausen, The Multi-Agent Transport Simulation MATSim. London: Ubiquity Press, 2016.

[11] J. Bischoff and M. Maciejewski, "Autonomous taxicabs in berlin a spatiotemporal analysis of service performance," Transportation Research Procedia, vol. 19, pp. 176 - 186, 2016.

[12] M. Maciejewski and J. Bischoff, "Congestion effects of autonomous taxi fleets," Transport, vol. 33, pp. 1-10, 092017.

[13] J. Bischoff, M. Maciejewski, T. Schlenther, and K. Nagel, "Autonomous vehicles and their impact on parking search," IEEE Intelligent Transportation Systems Magazine, pp. 1-1, 102018.

[14] S. Hörl, C. Ruch, F. Becker, E. Frazzoli, and K. W. Axhausen, "Fleet operational policies for automated mobility: a simulation assessment for Zurich," Under Review, 2019.

[15] P. M. Bösch, F. Becker, H. Becker, and K. W. Axhausen, "Cost-based analysis of autonomous mobility services," Transport Policy, vol. 64, pp. $76-91,2018$

[16] J. Wen, Y. X. Chen, N. Nassir, and J. Zhao, "Transit-oriented autonomous vehicle operation with integrated demand-supply interaction," Transportation Research Part C: Emerging Technologies, vol. 97 , pp. $216-234,2018$.

[17] F. Dandl, B. Bracher, and K. Bogenberger, "Microsimulation of an autonomous taxi-system in munich," 062017.

[18] F. Dandl and K. Bogenberger, "Comparing future autonomous electric taxis with an existing free-floating carsharing system," IEEE Transactions on Intelligent Transportation Systems, pp. 1-11, 2018.

[19] L. M. Martinez and J. M. Viegas, "Assessing the impacts of deploying a shared self-driving urban mobility system: An agent-based model applied to the city of lisbon, portugal," International Journal of Transportation Science and Technology, vol. 6, no. 1, pp. 13 - 27, 2017.
[20] A. Poulhès and J. Berrada, "User assignment in a smart vehicles network: dynamic modelling as an agent-based model," Transportation Research Procedia, vol. 27, pp. 865 - 872, 2017.

[21] J. Berrada, I. Andreasson, W. Burghout, and F. Leurent, "Demand modeling of autonomous shared taxis mixed with scheduled transit," 98th Annual Meeting of the Transportation Research Board, 2019.

[22] J. Kamel, R. Vosooghi, J. Puchinger, F. Ksontini, and G. Sirin, "Exploring the Impact of User Preferences on Shared Autonomous Vehicle Modal Split: A Multi-Agent Simulation Approach," Transportation Research Procedia, vol. 37, pp. 115-122, 2018.

[23] R. Vosooghi, J. Kamel, J. Puchinger, V. Leblond, and M. Jankovic, "Robo-taxi service fleet sizing: Assessing the impact of user trust and willingness to use," 98th Annual Meeting of the Transportation Research Board, 2019.

[24] S. Hörl, M. Balac, and K. W. Axhausen, "A first look at bridging discrete choice modeling and agent-based microsimulation in matsim," Procedia Computer Science, vol. 130, pp. 900 - 907, 2018.

[25] — , "Pairing discrete mode choice models and agent-based transport simulation with MATSim," 98th Annual Meeting of the Transportation Research Board, 2019.

[26] K. E. Train, Discrete choice methods with simulation. Cambridge university press, 2009.

[27] S. Hörl, "Agent-based simulation of autonomous taxi services with dynamic demand responses," Procedia Computer Science, vol. 109, pp. $899-904,2017$.

[28] M. Maciejewski, J. Bischoff, S. Hörl, and K. Nagel, "Towards a testbed for dynamic vehicle routing algorithms," in Highlights of Practical Applications of Cyber-Physical Multi-Agent Systems, J. Bajo, Z. Vale, K. Hallenborg, A. P. Rocha, P. Mathieu, P. Pawlewski, E. Del Val, P. Novais, F. Lopes, N. D. Duque Méndez, V. Julián, and J. Holmgren, Eds. Cham: Springer International Publishing, 2017, pp. 69-79.

[29] C. Ruch, S. Hörl, and E. Frazzoli, "AMoDeus, a Simulation-Based Testbed for Autonomous Mobility-on-Demand Systems," 21st International Conference on Intelligent Transportation Systems (ITSC), 2018.

[30] F. Poletti, P. M. Bsch, F. Ciari, and K. W. Axhausen, "Public transit route mapping for large-scale multimodal networks," vol. 6, no. 9, p. 268, 2017-09.

[31] D. Meunier and E. Quinet, "Value of time estimations in cost benefit analysis: The french experience," Transportation Research Procedia, vol. 8, pp. $62-71,2015$.

[32] S. Hörl, M. Balac, and K. W. Axhausen, "An agent-based transport model for Île-de-France and Paris," Arbeitsberichte Verkehrs-und Raumplanung, 2019.

[33] H. Becker, F. Becker, R. Abe, S. Bekhor, P. F. Belgiawan, J. Compostella, E. Frazzoli, L. M. Fulton, N. Garrick, D. Guggisberg Bicudo et al., "Impact of vehicle automation and electric propulsion on production costs for mobility services worldwide," Arbeitsberichte Verkehrs-und Raumplanung, vol. 1371, 2018.

[34] R. Haslebacher, "Intermodal Routing in MATSim Applied to SBB Green Class," Master's thesis, ETH Zurich, 2018. 